LBNL-40497

\title{
A microscopic calculation of secondary Drell-Yan production in heavy ion collisions
}

\author{
C. Spieles, L. Gerland, N. Hammon, M. Bleicher, S.A. Bass, H. Stöcker, W. Greiner \\ Institut für Theoretische Physik, J. W. Goethe-Universität, 60054 Frankfurt am Main, Germany \\ C. Lourenço \\ CERN PPE, CH-1211 Geneva 23, Switzerland \\ R. Vogt \\ Nuclear Science Division, LBNL, Berkeley, CA 94720, USA \\ Physics Department, University of California, Davis, CA 95616, USA
}

\begin{abstract}
A study of secondary Drell-Yan production in nuclear collisions is presented for SPS energies. In addition to the lepton pairs produced in the initial collisions of the projectile and target nucleons, we consider the potentially high dilepton yield from hard valence antiquarks in produced mesons and antibaryons. We calculate the secondary Drell-Yan contributions taking the collision spectrum of hadrons from the microscopic model URQMD. The contributions from meson-baryon interactions, small in hadron-nucleus interactions, are found to be substantial in nucleus-nucleus collisions at low dilepton masses. Preresonance collisions of partons may further increase the yields.
\end{abstract}

Typeset using REVTEX 


\section{INTRODUCTION}

A unique experimental signature from the early stage of violent heavy ion collisions is provided by dilepton radiation. The (thermal) radiation of leptons is highest when the energy density has reached a maximum. Since leptons are insensitive to strong interactions, the signal is not distorted during the further evolution of the system. Recent reports on intermediate [1] and high mass [2] muon pairs produced in heavy ion collisions have attracted much attention. The suppression of the $\mathrm{J} / \psi$ signal, as extracted from NA38/NA50 measurements, might indicate that deconfinement has been reached in central $\mathrm{Pb}+\mathrm{Pb}$ reactions [3]. In the mass region below the $\rho$-meson, on the other hand, the measured $\mathrm{e}^{+} \mathrm{e}^{-}$pair spectrum is enhanced relative to the expected 'cocktail' of hadronic decays [4]. A good understanding of the continuum dilepton spectrum, in the relevant invariant mass window, is a prerequisite for the correct interpretation of the data.

Rate estimates of Drell-Yan pair production [5] have been performed systematically for different systems, energies, and invariant masses [6]. They are essentially based on extrapolations of $\mathrm{p}+\mathrm{A}$ reactions where a linear scaling with $\mathrm{A}$ is observed for the Drell-Yan production cross section [0]:

$$
\sigma_{\mathrm{pA}}=\sigma_{0} \mathrm{~A}
$$

This linear scaling can be understood in the Glauber picture of the hadron-nucleus cross section, constructed at high energies using the AGK cutting rules [8]. (For a simple dynamical picture of this effect, see [9].)

A number of different theoretical frameworks [10 15 have been used to explore the degree to which the hot matter produced in ultrarelativistic heavy ion collisions contributes to the total dilepton radiation. Dilepton emission from thermal sources such as a quark-gluon plasma or hadron gas, whether in or out of equilibrium, cannot be neglected relative to nucleon-nucleon contributions to the Drell-Yan process for masses below the $\mathrm{J} / \psi$ peak at least at higher energies than those presently available. Another source of dileptons which 
has not been fully explored is Drell-Yan production by interactions between produced, or secondary, hadrons. Unless these secondary dilepton sources are understood, the thermal contributions cannot be reliably extracted. In this paper, we study possible refinements to Drell-Yan production in nucleus-nucleus collisions from secondary hadron interactions at current fixed-target energies. It is shown that these secondary collisions can serve as an important source of $m \sim 2 \mathrm{GeV}$ dileptons due to the availability of valence antiquarks in mesons and antibaryons.

\section{THE MODEL}

In order to investigate secondary dilepton production at SPS energies円 a microscopic hadronic transport code, URQMD [16], is employed to obtain a realistic collision spectrum of secondary hadrons. The differential Drell-Yan cross section is computed at leading order (LO) using the standard equation [17]:

$$
\frac{\mathrm{d}^{2} \sigma}{\mathrm{d} m^{2} \mathrm{~d} y}(\mathrm{AB} \rightarrow l \bar{l} X)=\frac{4 \pi \alpha^{2}}{9 m^{2} s} \sum_{q} e_{q}^{2}\left[q^{\mathrm{A}}\left(x_{\mathrm{A}}, m^{2}\right) \bar{q}^{\mathrm{B}}\left(x_{\mathrm{B}}, m^{2}\right)+\bar{q}^{\mathrm{A}}\left(x_{\mathrm{A}}, m^{2}\right) q^{\mathrm{B}}\left(x_{\mathrm{B}}, m^{2}\right)\right]
$$

where $q\left(x, m^{2}\right)$ and $\bar{q}\left(x, m^{2}\right)$ denote the quark and antiquark densities, $\sqrt{s}$ is the centerof-mass energy of the colliding hadrons, $m$ is the invariant mass of the lepton pair, $x_{\mathrm{A}}=$ $\sqrt{\tau} e^{y}$ and $x_{\mathrm{B}}=\sqrt{\tau} e^{-y}$ with $\tau=m^{2} / s$, and $y$ is the dilepton rapidity in the cms frame. The convolution of the parton distributions is weighted by the square of the charge of the annihilating quarks, $e_{q}$. Since the calculation is performed at leading order only, the result is multiplied by a "K-factor" [18. Although the ratio between the calculated next-to-leading order (NLO) and LO Drell-Yan cross sections is a function of $m^{2}$ and $y$, the dependence is

\footnotetext{
${ }^{1}$ At RHIC and LHC energies the heavy flavour (c and b) combinatorial decays are expected to dominate the dilepton continuum [15]. Moreover, the nuclear parton distribution functions at such small $x$ values are still quite uncertain. Therefore, we will not report any high energy calculations in this paper.
} 
small, $\sim 25 \%$ for $m>1.5 \mathrm{GeV}$ and $0<y<1$ at $\sqrt{s}=19.4 \mathrm{GeV}$. Thus, for simplicity, we have used a constant $\mathrm{K}$ factor. This is reasonable considering the accuracy of the present experimental data which does not reveal any strong mass dependence of the experimental $\mathrm{K}$ factor 7 (the ratio between the measured data and the LO calculation).

According to the URQMD hadronic model, most of the interacting particles in the early stage of the reaction are baryon and meson resonances. In this study, if two hadrons have the same quark content, their parton distributions are assumed to be equivalent, e.g. the proton parton distributions are used for the $N^{*}(1440)$ and the pion parton distributions are used for the $\rho$. We have employed the GRV LO parametrizations of the parton distribution functions of nucleons [20] and pions [21], since they provide a consistent set for both species (same $\Lambda_{\mathrm{QCD}}$ and similar initial scale $\left.\mu_{0}\right)$. The lepton pair production cross section is calculated for each hadron-hadron collision and weighted by the inverse of the total hadron-hadron cross section. The distributions from these elementary hh-collisions are then summed.

It is clear that in pion-nucleon, pion-pion and antinucleon-nucleon collisions, valence quark-antiquark annihilation can play a significant role in the Drell-Yan process. Figure 1 shows the parton distribution functions of protons [20] and pions [21] from the GRV LO parametrization, for two different values of $m^{2}$. For $x \gtrsim 0.05$ the probability to find an antiquark is much higher in a pion than in a nucleon. The pion-nucleon cross sections are consequently higher than the nucleon-nucleon cross sections, especially when $m / \sqrt{s} \gtrsim$ 0.1. (See [17.) On the other hand, in heavy ion collisions the typical meson-baryon and antibaryon-baryon interactions are certainly less energetic than the primary nucleon-nucleon collisions.

While the usual Drell-Yan calculations of nucleus-nucleus collisions only consider interactions of the initial nucleons, in the present scenario four different processes may con-

\footnotetext{
${ }^{2}$ This situation might change in the near future when the very high statistics Drell-Yan differential cross sections of E866 [19] become available.
} 
tribute: baryon-baryon, meson-baryon (and meson-antibaryon), meson-meson ad baryonantibaryon interactions. Thus, it is interesting to estimate the relative importance of these contributions to the total Drell-Yan cross section.

The typical scaling of the Drell-Yan cross section with target mass observed in hadronnucleus collisions, accounted for here, assumes an equal probability for Drell-Yan pair production in each nucleon-nucleon interaction. In addition, in a separate microscopic simulation the meson-baryon, meson-meson and baryon-antibaryon collisions, involving newly produced hadrons, are calculated within the URQMD transport model. In order to conserve energy and momentum, the energy loss of baryons which have already interacted is taken into account in these secondary interactions.

For simplicity, the produced hadrons follow straight-line trajectories across the reaction zone without energy loss ( $0^{\circ}$-scattering, only elastic interactions). The center of mass energy of these collisions is taken directly from the fully dynamical evolution of all hadrons within the URQMD simulation. In the usual cascade prescription, leading hadrons, containing valence quarks from the incident nucleon, can interact within their formation time with a reduced but finite cross section. Since the constituent antiquark content is much more relevant for the Drell-Yan process, we only consider collisions of formed hadrons. The formation time of produced hadrons, on average $\tau_{F} \approx 1 \mathrm{fm} / \mathrm{c}$, is determined by the string fragmentation prescription used in URQMD [16] (defined as the first crossing point of the quark and antiquark or quark and diquark which form the hadron). The exact formation time depends on the mass and momentum of the produced hadron. By imposing a minimum hadron formation time, we effectively neglect any possible annihilation of produced partons

\footnotetext{
${ }^{3}$ The Drell-Yan process in meson-meson interactions should not be confused with processes such as $\pi^{+} \pi^{-} \rightarrow l^{+} l^{-}$where the whole meson is involved in the annihilation rather than a single parton. In any case, dileptons from pion annihilations are not expected to make an important contribution to the intermediate mass region we are concerned with here.
} 
which have not yet hadronized ("preresonance" scattering). In Sec. VI we return to this problem.

\section{THE EFFECT OF A FLAVOUR ASYMMETRIC SEA}

Important consequences for the sea quark distributions have recently been discussed: modifications at small $x$ and the violation of the Gottfried sum rule [22]. Two effects are primarily important at low $x$. The HERA data has shown a steep rise in the proton structure function $F_{2}$ for $x \lesssim 0.01$ [23]. Additionally, the sea quark and gluon distributions can be significantly modified in nuclear targets [24]. The observed low $x$ shadowing and the low $x$ rise in the parton sea distributions are not relevant for SPS energies where $0.077<x<0.52$ for $1.5<m<10 \mathrm{GeV}$ at midrapidity. At higher energies, such as those at RHIC and LHC, these effects cannot be neglected. However, as we now describe, the violation of the Gottfried sum rule has important consequences at the SPS energies.

The Gottfried sum rule defines the difference between the proton and neutron structure functions [22]

$$
\begin{aligned}
I_{G S R} & =\Sigma(0,1)=\int_{0}^{1} \frac{d x}{x}\left(F_{2}^{p}-F_{2}^{n}\right) \\
& =\frac{1}{3} \int_{0}^{1} d x\left(u_{v}-d_{v}\right)+\frac{2}{3} \int_{0}^{1} d x(\bar{u}-\bar{d})=\frac{1}{3} \text { if } \bar{u}=\bar{d} .
\end{aligned}
$$

Since the neutron distributions are not directly measured, $F_{2}^{n}$ is obtained assuming that $u^{p}\left(x, m^{2}\right)=d^{n}\left(x, m^{2}\right)$ and $\bar{u}^{p}\left(x, m^{2}\right)=\bar{d}^{n}\left(x, m^{2}\right)$. All global structure function analyses prior to 1992 assumed that the light-quark sea distributions are flavour independent, $\bar{u}\left(x, m^{2}\right)=\bar{d}\left(x, m^{2}\right)$. On the contrary, based on their $F_{2}^{n} / F_{2}^{p}$ measurements, NMC found that [25]

$$
\Sigma(0.004,0.8)=\int_{0.004}^{0.8} \frac{d x}{x}\left(F_{2}^{p}-F_{2}^{n}\right)=0.227 \pm 0.007 \quad \text { (stat.) } \pm 0.014 \quad \text { (syst.) }
$$

at $m^{2}=4 \mathrm{GeV}^{2}$. A straightforward comparison implies that $\bar{d}>\bar{u}$. These results have been confirmed by $\mathrm{p}+\mathrm{p}$ and $\mathrm{p}+\mathrm{d}$ asymmetry studies of Drell-Yan production by NA51 26] and 
by preliminary results from E866 [19]. The lack of a Regge $f-A_{2}$ exchange degeneracy also leads one to expect that 27]

$$
\Delta \equiv \bar{d}-\bar{u} \sim x^{-\alpha_{R}}
$$

at small $x$, where the Regge intercept is $\alpha_{R} \approx 0.5$.

With this in mind, we compare two versions of the GRV proton parametrizations, the flavour-symmetric set from 1992 [28] and the 1994 version [20] that explicitly takes into account the NA51 results by choosing

$$
\bar{u}=\frac{1}{2}[(\bar{u}+\bar{d})-\Delta], \quad \bar{d}=\frac{1}{2}[(\bar{u}+\bar{d})+\Delta]
$$

Another factor contributing to the difference between the two sets is the starting distribution used to obtain the two GRV valence distributions: the flavour symmetric KMRS B- [29] for GRV 92 and the flavour asymmetric MRS A [30] for GRV 94. These starting distributions differ at low $x$ and in the fitted value of $\Lambda_{\mathrm{QCD}}$ as well as in their treatment of the $\bar{u}$ and $\bar{d}$ distributions. To study the difference between these two GRV parametrizations, we evaluate the lepton pair production cross sections for $\mathrm{p}+\mathrm{p}, \mathrm{p}+\mathrm{n}$ and $\mathrm{n}+\mathrm{n}$ interactions. (Note that at $y=0, \mathrm{p}+\mathrm{n}=\mathrm{n}+\mathrm{p}$.) The ratios of cross sections from the GRV 94 relative to the GRV 92 parametrizations are shown in Fig. 2 las functions of $m$ at $y=0$ and $\sqrt{s}=15 \mathrm{GeV}$. The effect of the flavour asymmetric sea, most pronounced for $\mathrm{p}+\mathrm{n}$ and $\mathrm{n}+\mathrm{n}$ interactions, is clearly important. Inclusion of the flavour asymmetric sea decreases the isospin dependence of the Drell-Yan cross section. The isospin correction needed for $\mathrm{Pb}+\mathrm{Pb}$ interactions is 1.3 with the GRV $92 \mathrm{LO}$ set [2] and 1.03 with the GRV $94 \mathrm{LO}$ set. The smaller isospin correction is also found when MRS G distributions [31] are used.

Since neither corresponding $\bar{d}-\bar{u}$ data nor parton distributions with a flavour asymmetric sea exist for pions, we use the flavour symmetric GRV distributions for the pion [21] in our meson interactions. One might suppose the asymmetry to be less pronounced for the pion since the $\pi^{+}(u \bar{d})$ and $\pi^{-}(\bar{u} d)$ each contain a $u$ and a $d$ quark or antiquark. 


\section{PROTON-NUCLEUS AND PION-NUCLEUS REACTIONS}

Figure 3 shows the mass spectrum of dimuons in $\mathrm{p}+\mathrm{Pt}$ collisions at $p_{\text {lab }}=400 \mathrm{GeV} / \mathrm{c}$ for three different longitudinal momentum windows. Our calculation with the GRV 94 parton distributions provides a reasonable description of the NA3 data 32 with $\mathrm{K}=1.5$. In addition we have compared the model calculation with $\mathrm{p}+\mathrm{Cu}$ collisions at $p_{\text {lab }}=800 \mathrm{GeV} / \mathrm{c}$ [33] and found equally good agreement. In fact, the Drell-Yan mass and $x_{F}$ double differential cross sections measured by NA3 [32, E605 33] and E772 34 experiments, are all in good agreement with each other, as shown in Ref. 355.

The proton-nucleus calculations reveal that the contribution from secondary collisions is less than $10 \%$ of the dilepton yield for masses $m>1.5 \mathrm{GeV}$. The relative importance of secondary contributions decreases with increasing $x_{F}$. At $x_{F}=0.325$ and $x_{F}=0.625$, the original Glauber type calculation is increased by less than 1\%, even at the lowest masses.

The dimuon spectrum produced in $\pi^{-}+W$ reactions, at $p_{\text {lab }}=125 \mathrm{GeV} / \mathrm{c}$, as measured by the E537 experiment [36], is presented in Fig. 1f for a central and a forward $x_{F}$ bin. The results of our leading order calculations, scaled by a $\mathrm{K}$ factor of 2 , are shown. A different $\mathrm{K}$ factor relative to the value used in the proton-induced reactions is needed since we use the GRV 92 pion set with the GRV 94 proton set. Although the measurements have large uncertainties, the agreement between our calculations and the data supports the validity of our treatment of secondary meson-baryon collisions.

\section{NUCLEUS-NUCLEUS COLLISIONS}

Figure 5 shows the relative importance of the secondary dilepton production, compared to nucleon-nucleon collisions alone, for different projectile/target combinations. In the mass region around $2 \mathrm{GeV}$ the additional contributions are not negligible in nucleus-nucleus col-

lisions while the increase is less than $5 \%$ in the $\mathrm{p}+\mathrm{W}$ case. For masses above $3 \mathrm{GeV}$, the additional production beyond nucleon-nucleon collisions from our hadronic calculation is less 
than $10 \%$ in both $\mathrm{S}+\mathrm{U}$ and $\mathrm{Pb}+\mathrm{Pb}$ interactions. Evidently the high mass dilepton yield is not significantly enhanced by the relatively low energy secondary scatterings. At masses below $2 \mathrm{GeV}$, on the contrary, it appears that the contribution from collisions of newly produced particles cannot be neglected. At masses around $1.5 \mathrm{GeV}$, an enhancement of $25 \%$ is expected in $\mathrm{S}+\mathrm{U}$ interactions and $45 \%$ in $\mathrm{Pb}+\mathrm{Pb}$ interactions. Clearly, the importance of secondary contributions to the Drell-Yan cross section increases with the system size. Note, however, that this enhancement is insufficient to account for the discrepancy reported in Ref. [1] between the data and the "expected sources".

Although the perturbative QCD calculation of dilepton production at $2 \mathrm{GeV}$ and below can only be used as a qualitative guideline, it is clear that the low mass dilepton continuum in nuclear collisions can be enhanced relative to a linear superposition of nucleon-nucleon interactions. At least part of the observed enhancement of muon pairs at intermediate and low masses [1] might be caused by this previously neglected hadronic source. The perturbative calculation is definitely unreliable for masses below $1 \mathrm{GeV}$ where a substantial increase in electron pair production in nucleus-nucleus collisions has been observed [4]. To emphasize the theoretical uncertainty of the perturbative calculation for masses below $2 \mathrm{GeV}$, in Fig. 5 and in the following figures, we use thinner lines in that region.

The relative contributions to the calculated dilepton mass spectra at $y_{\mathrm{cms}}=0.5$ in central $\mathrm{S}+\mathrm{U}$ and $\mathrm{Pb}+\mathrm{Pb}$ collisions are presented in Fig. 6. The decomposition of the secondary dilepton yield into each channel (meson-baryon, meson-meson, baryon-antibaryon) shows that by far the most important secondary contribution is from meson-baryon interactions. For masses above $\sim 3 \mathrm{GeV}$ they are virtually the only source of secondary dileptons. At midrapidity and for masses $\sim 1 \mathrm{GeV}$, they account for at least $90 \%$ of the secondary yield while meson-meson collisions contribute $\sim 10 \%$ and baryon-antibaryon collisions contribute less than $1 \%$. 


\section{PRIMORDIAL SOURCES OF LEPTON PAIRS AND FORMATION TIME}

The standard Drell-Yan process corresponds to the interaction of fully formed hadrons. However, it was shown [3] that, during the early stages of the system evolution, partons can scatter and annihilate before they have come on mass-shell f. Thus, we postulate the existence of another dilepton source: the annihilation of quarks and antiquarks which are not yet bound in a color-singlet hadron.

To estimate the importance of these "primordial" or "preresonance" $q \bar{q}$ annihilations, we have calculated the contribution of such processes assuming that the asymptotic parton distribution functions are also valid for the primordial states. This assumption is distinct from thermal dilepton production where the quark and antiquark are in a thermal environment, in or out of equilibrium, with temperature-dependent parton densities. We thus relax the restriction that the partons can only interact after they have hadronized. This is done very simply in our microscopic cascade calculation by decreasing the formation time of the produced hadrons, $\tau_{F}$, from the "default" value of around $1 \mathrm{fm} / c$.

The importance of this primordial (preresonance) contribution to the dilepton mass spectra is shown in Fig. 7 . We see that when we set $\tau_{F}=0$, the secondary sources of dileptons become much more important than previously indicated. Indeed, the secondary dilepton yield increases by a factor of $\sim 5$ at all masses compared to the calculations with the default $\tau_{F} \approx 1 \mathrm{fm} / c$. These primordial interactions also affect $\mathrm{p}+\mathrm{A}$ reactions, enhancing the secondary contribution. For example, the dilepton yield at $m=4.5 \mathrm{GeV}$ and $x_{F}=0.025$ shown in Fig. 3 increases by $25 \%$ when $\tau_{F}=0$. Nevertheless, good agreement with the NA3 data is retained.

In Fig. 7 we have also included a calculation with an intermediate formation time for the produced hadrons, $\tau_{F}=0.5 \mathrm{fm} / \mathrm{c}$, in order to study the sensitivity of the calculations

\footnotetext{
${ }^{4}$ There are some similarities between the off-shell interactions of the primordial partons and the soft parton annihilation model proposed to explain the "anomalous" low mass lepton pairs [37.
} 
to this (model dependent) parameter. As we might expect, the reduced average formation time leads to an increased dilepton yield due to the larger number of possible hadronhadron interactions. With this value of the formation time, the enhancement in the range $1.5<m<2 \mathrm{GeV}$ shows quite good agreement with the intermediate mass data [1]. On the other hand, an infinite formation time, $\tau_{F}=\infty$, corresponds to the exclusion of all secondary interactions, in agreement with the Glauber-type Drell-Yan expectation. A recent work [38 has already pointed out the strong influence of the hadron formation time on dilepton production via $\pi^{+} \pi^{-}$annihilation.

\section{CONCLUSION}

Our study indicates that the lepton pair continuum cross section for masses up to $3 \mathrm{GeV}$ should not be interpreted solely in terms of Drell-Yan production in nucleon-nucleon interactions, even at SPS energies. The yield from secondary contributions depends strongly on the rapidity region and, of course, on the dilepton mass. Preresonance interactions are estimated to enhance the secondary yield by up to a factor of 5 .

Because meson-baryon interactions dominate secondary lepton pair production, the rela-

tive importance of secondary dilepton sources evidently increases linearly with the number of produced pions at these energies. It may thus be possible to distinguish the secondary yield from thermal dilepton sources which are expected to scale quadratically with the pion multiplicity. The multiplicity dependence also indicates that the dilepton yield from scattering of secondaries is likely to be considerably higher at RHIC and LHC energies.

\section{ACKNOWLEDGMENTS}

We would like to acknowledge interesting discussions with J. Schukraft. We thank the Institute for Nuclear Theory at the University of Washington, Seattle, where this work was initiated, for its kind hospitality. This work was supported by Graduiertenkolleg, Gesellschaft für Schwerionenforschung, Bundesministerium für Bildung und Forschung, 
Deutsche Forschungsgemeinschaft. The work of R. V. was supported in part by the Director, Office of Energy Research, Division of Nuclear Physics of the Office of High Energy and Nuclear Physics of the U. S. Department of Energy under Contract No. DE-AC03-76SF0098. 


\section{REFERENCES}

[1] C. Lourenço et al. (NA38 Coll.), Nucl. Phys. A566 (1994) 77c.

M.A. Mazzoni et al. (Helios-3 Coll.), Nucl. Phys. A566 (1994) 95c.

C. Lourenço, in Proceedings of the Hirschegg '95 Workshop, p.163 (CERN-PPE/95-72).

I. Kralik, in Proceedings of the Hirschegg '95 Workshop, p.143.

E. Scomparin et al. (NA50 Coll.), Nucl. Phys. A610 (1996) 331c.

[2] M. Gonin et al. (NA50 Coll.), Nucl. Phys. A610 (1996) 404c.

F. Fleuret et al. (NA50 Coll.), in Proceedings of the 32nd Rencontres de Moriond, QCD and High Energy Hadronic Interactions, Les Arcs, France, 1997.

[3] D. Kharzeev, Proc. of the International School of Physics, "Enrico Fermi", Course 80: Selected Topics in Nonperturbative QCD, Varenna, Italy, p. 105 (nucl-th/9601029).

H. Satz, in Proceedings of the 31st Rencontres de Moriond, QCD and High Energy

Hadronic Interactions, Les Arcs, France, 1996, p.419 (CERN-TH/96-118).

D. Kharzeev, C. Lourenço, M. Nardi and H. Satz, Z. Phys. C74 (1997) 307.

[4] Th. Ullrich et al. (CERES Coll.), Nucl. Phys. A610 (1996) 317.

A. Drees, in Proceedings of the Hirschegg '97 Workshop, p.178.

[5] S.D. Drell and T.-M. Yan, Phys. Rev. Lett. 25 (1970) 316.

[6] R. Vogt, Atomic Data and Nuclear Data Tables 50 (1992) 343.

[7] D.M. Alde et al. (E772 Coll.), Phys. Rev. Lett. 64 (1990) 2479.

[8] V. Abramovskij, V.N. Gribov and O.V. Kancheli, Yad. Phys. 18 (1973) 595 [Sov. J. Nucl. Phys. 18 (1974) 308].

[9] G.R. Farrar, Phys. Lett. B56 (1975) 185.

[10] J. Kapusta, L. McLerran and D.K. Srivastava, Phys. Lett. B283 (1992) 145.

[11] B. Kämpfer and O.P. Pavlenko, Phys. Lett. B289 (1992) 127. 
[12] A. Dumitru, D.H. Rischke, T. Schönfeld, L. Winckelmann, H. Stöcker and W. Greiner, Phys. Rev. Lett. 70 (1993) 2860.

[13] L.A. Winckelmann, H. Sorge, H. Stöcker and W. Greiner, Phys. Rev. C51 (1995) R9.

[14] K. Geiger and J.I. Kapusta, Phys. Rev. Lett. 70 (1993) 1920.

[15] S. Gavin, P.L. McGaughey, P.V. Ruuskanen and R. Vogt, Phys. Rev. C54 (1996) 2606.

[16] S.A. Bass, M. Bleicher, M. Brandstetter, C. Ernst, L. Gerland, C. Hartnack, J. Konopka, S. Soff, C. Spieles, H. Weber, L.A. Winckelmann, J. Aichelin, N. Amelin, H. Stöcker and W. Greiner: URQMD, source code and technical documentation, to be published; S.A. Bass et al., in Proc. of the International Conference on Nuclear Physics at the Turn of the Millennium, Wilderness, South Africa, 1996, p.399.

L.A. Winckelmann et al., Nucl. Phys. A610 (1996) 116c.

[17] F. Halzen and D.M. Scott, Phys. Rev. D18 (1978) 3378.

[18] S. Gavin, S. Gupta, R. Kauffman, P.V. Ruuskanen, D.K. Srivastava and R.L. Thews, Int. J. Mod. Phys. A10 (1995) 2961.

[19] E. Hawker et al. (E866 Coll.), in Proceedings of the 32nd Rencontres de Moriond, QCD and High Energy Hadronic Interactions, Les Arcs, France, 1997,

[20] M. Glück, E. Reya, A. Vogt, Z. Phys. C67 (1995) 433.

[21] M. Glück, E. Reya, A. Vogt, Z. Phys. C53 (1992) 651.

[22] K. Gottfried, Phys. Rev. Lett. 18 (1967) 1174.

[23] M. Derrick et al. (ZEUS Coll.), Phys. Lett. B316 (1993) 515.

[24] M. Arneodo, Phys. Rep. 240 (1994) 301.

[25] P. Amaudruz et al. (NMC Coll.), Phys. Rev. Lett. 66 (1991) 2712.

[26] A. Baldit et al. (NA51 Coll.), Phys. Lett. B332 (1994) 244. 
[27] A.D. Martin, R.G. Roberts and W.J. Stirling, Phys. Rev. D47 (1993) 867.

[28] M. Glück, E. Reya, A. Vogt, Z. Phys. C53 (1992) 127.

[29] J. Kwiecinski, A.D. Martin, R.G. Roberts and W.J. Stirling, Phys. Rev. D42 (1990) 3645 .

[30] A.D. Martin, R.G. Roberts and W.J. Stirling, Phys. Rev. D50 (1994) 6734.

[31] A.D. Martin, R.G. Roberts and W.J. Stirling, Phys. Lett. B354 (1995) 155.

[32] J. Badier et al. (NA3 Coll.), Z. Phys. C26 (1984) 489.

[33] G. Moreno et al. (E605 Coll.), Phys. Rev. D43 (1991) 2815.

[34] P.L. McGaughey et al. (E772 Coll.), Phys. Rev. D50 (1994) 3038.

[35] P.L. McGaughey, Nucl. Phys. A610 (1996) 394c.

[36] E. Anassontzis et al., Phys. Rev. D38 (1988) 1377.

[37] J.D. Bjorken and H. Weisberg, Phys. Rev. D13 (1976) 1405; V. Cerny, P. Lichard and J. Pišút, Z. Phys. C31 (1986) 163.

[38] P. Filip and J. Pišút, CU-TH-97-05, nucl-th/9705051 and references therein. 


\section{FIGURES}

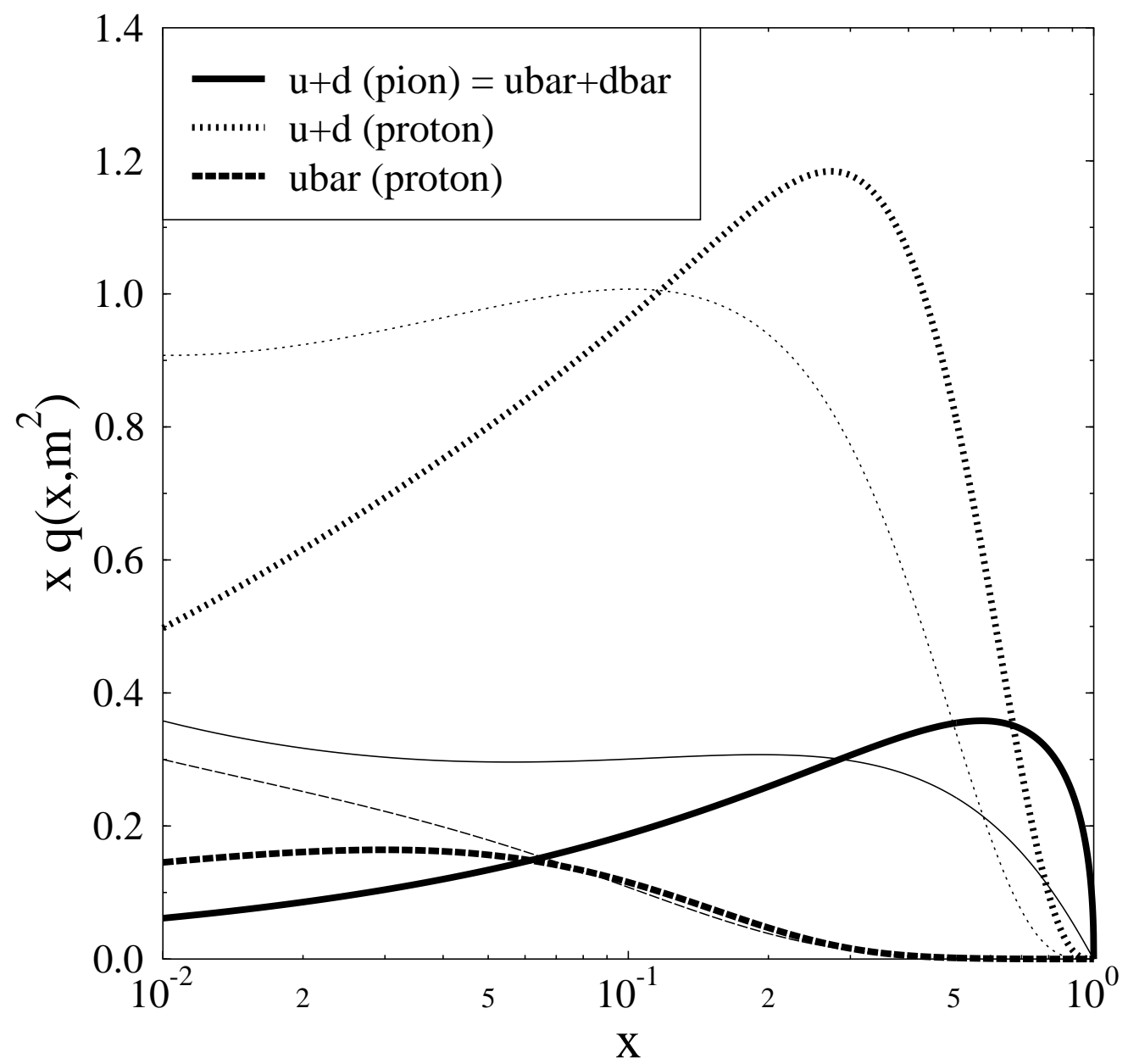

FIG. 1. The GRV LO parton distribution functions of protons [20] and pions [21] for valence and sea quarks. We show the values for $(u+d)$ quarks in the proton (dotted) and in the pion (full lines), and the values for $\bar{u}$ quarks in the proton (dashed lines). The thick lines indicate the distributions for $m^{2} \approx 0.3 \mathrm{GeV}^{2}$, the thin lines those for $m^{2}=9 \mathrm{GeV}^{2}$. Note the hard valence antiquark distribution in the pion. The valence antiquark distributions in the antibaryon correspond to the valence quark distributions in the baryons. 


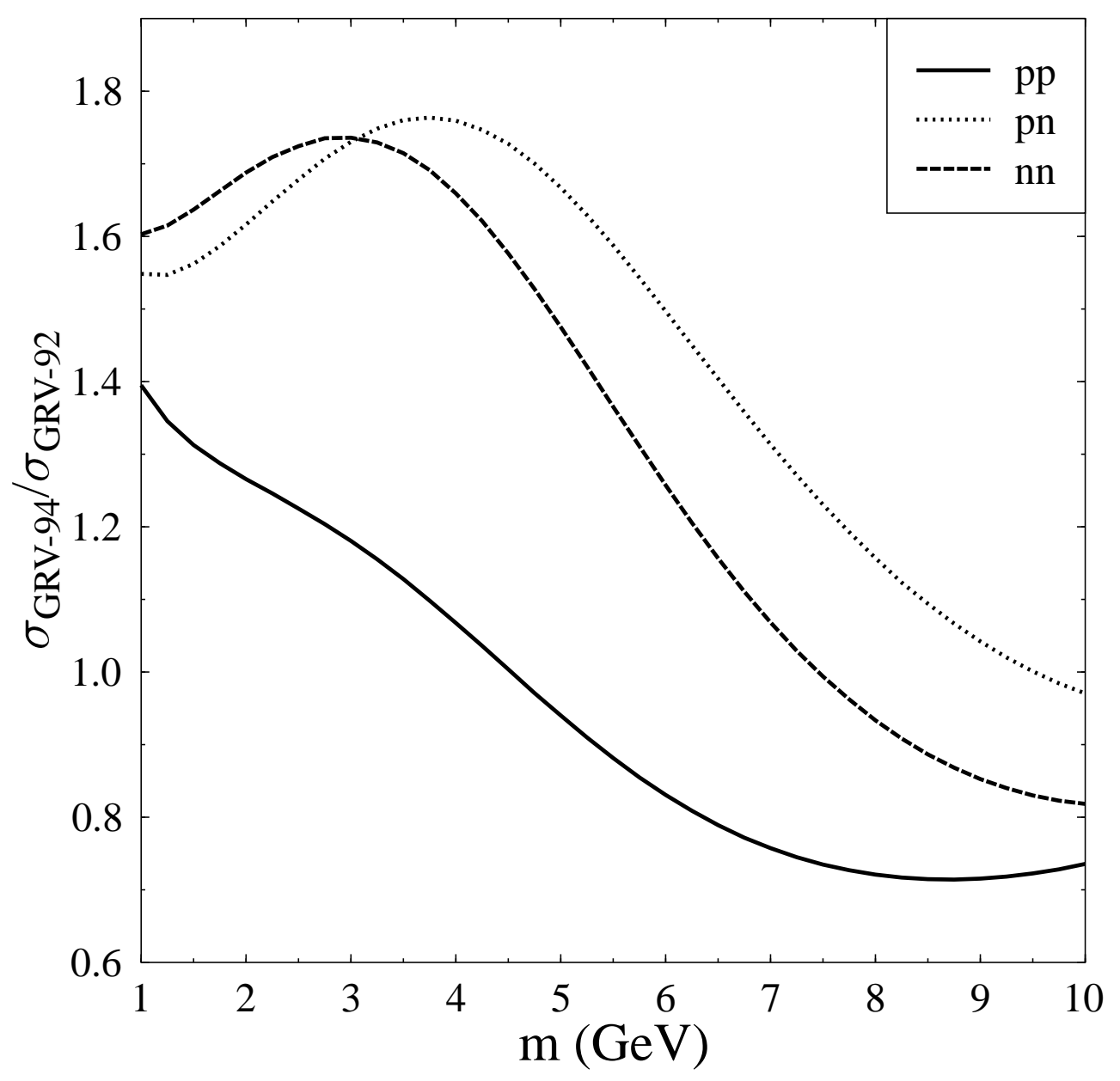

FIG. 2. Drell-Yan cross section calculated with the recent GRV 94 LO parametrizations 20] where $\bar{u} \neq \bar{d}$, relative to the calculation done with the GRV 92 LO set [28] which assumes a symmetric sea. Notice that the $\mathrm{K}$ factor to be applied to these calculations is 2 for GRV LO 92 and 1.5 to GRV LO 94. 


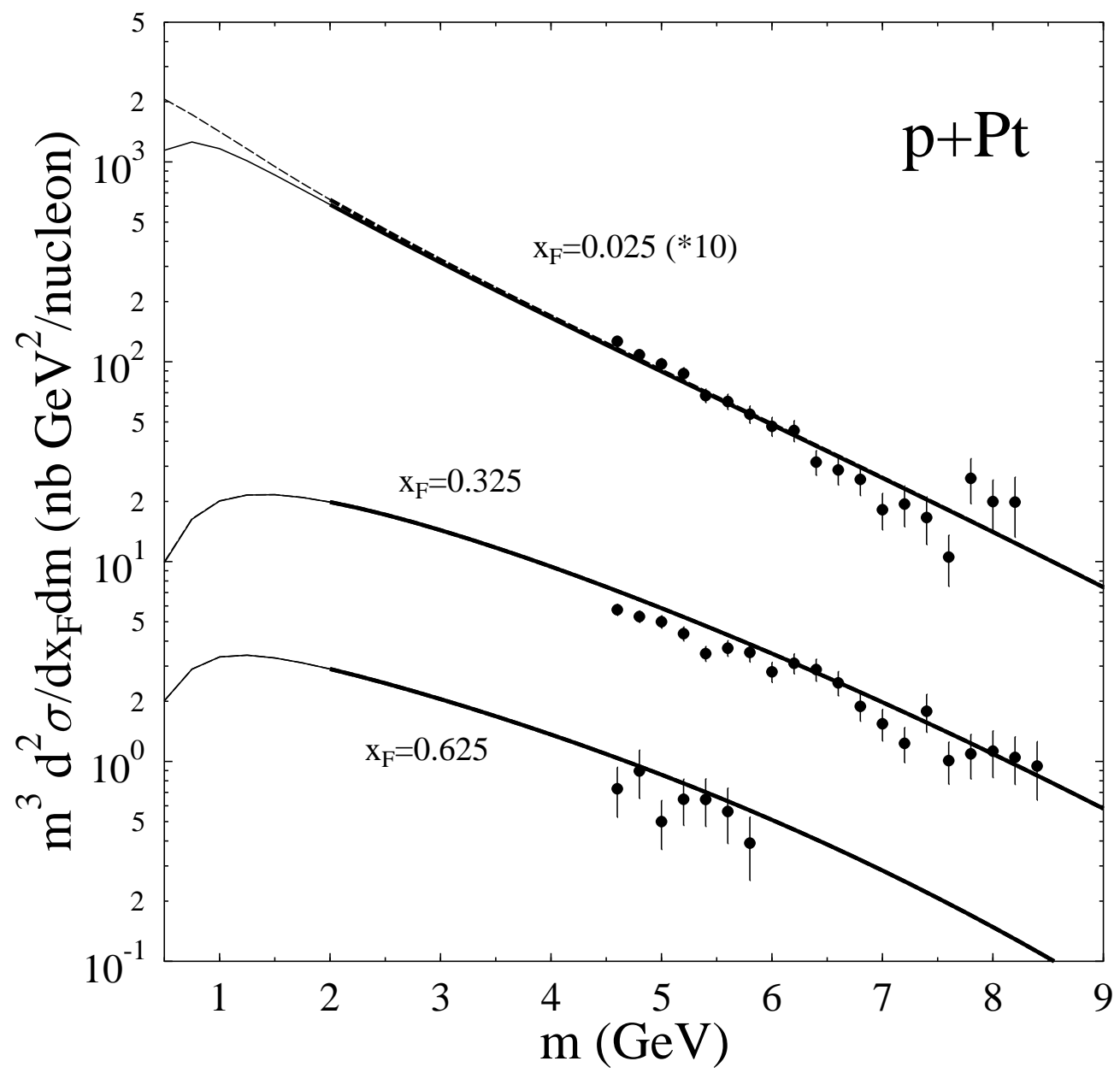

FIG. 3. Dilepton mass distributions from $\mathrm{p}+\mathrm{Pt}$ collisions at $p_{\text {lab }}=400 \mathrm{GeV} / \mathrm{c}$ calculated for three $x_{F}$ values. The contribution of baryon-baryon collisions is shown (solid line) separately from the total spectra (dashed line) which includes secondaries. The data points are from experiment NA3 [32]. We use a K factor of 1.5 for NN collisions (calculated with the GRV 94 nucleon PDFs) and of 2 for pion induced reactions (calculated with the GRV 92 pion PDFs). We use thinner lines for $m<2 \mathrm{GeV}$ to emphasize the relatively large theoretical uncertainty in the calculation. 


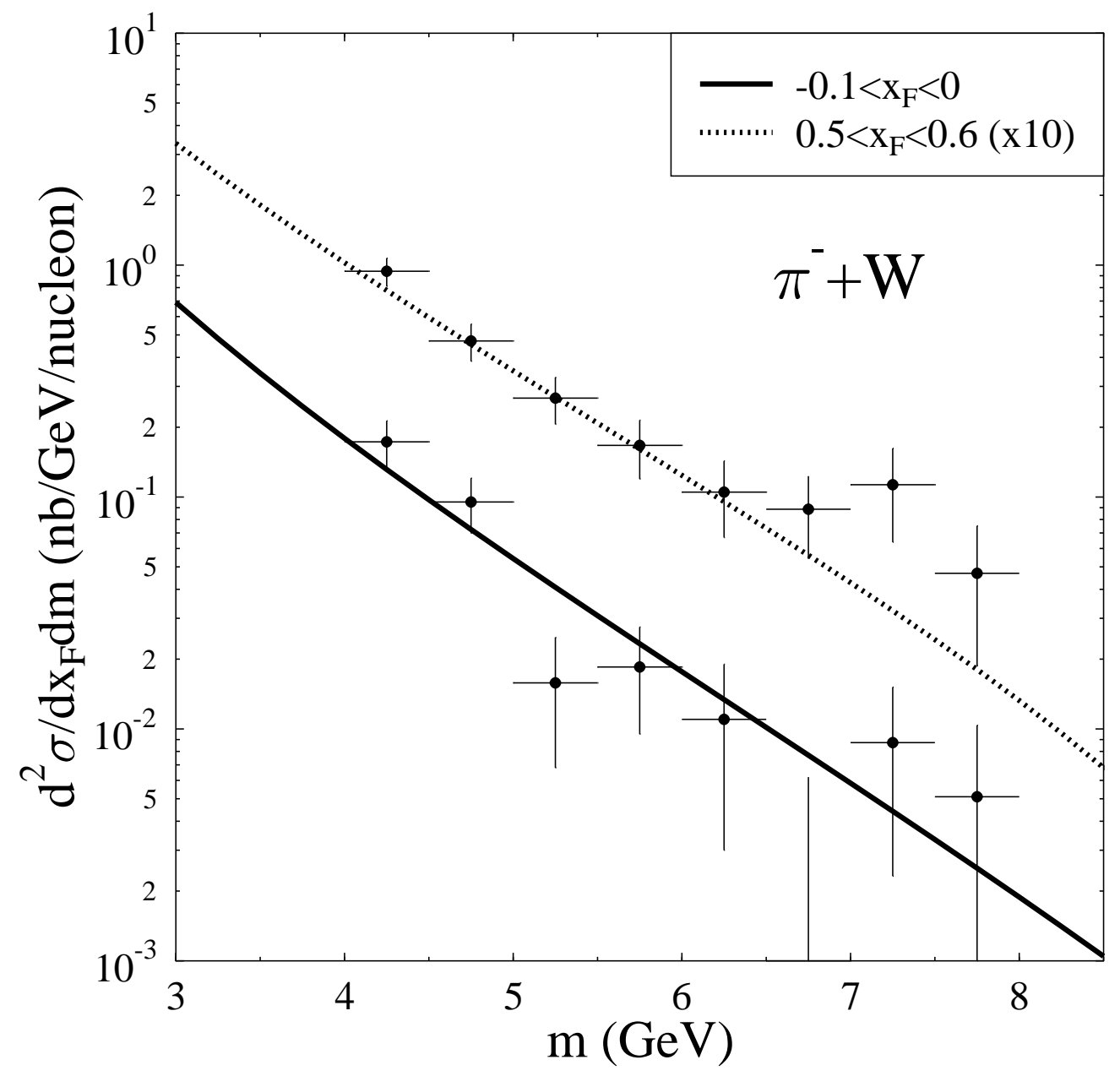

FIG. 4. Mass spectrum of dileptons for two $x_{F}$ bins in $\pi^{-}+W$ collisions at $p_{\text {lab }}=125 \mathrm{GeV} / \mathrm{c}$. We show our leading order calculations with data from experiment FNAL-537 [36]. We use a K factor of 2 for these pion induced reactions. 


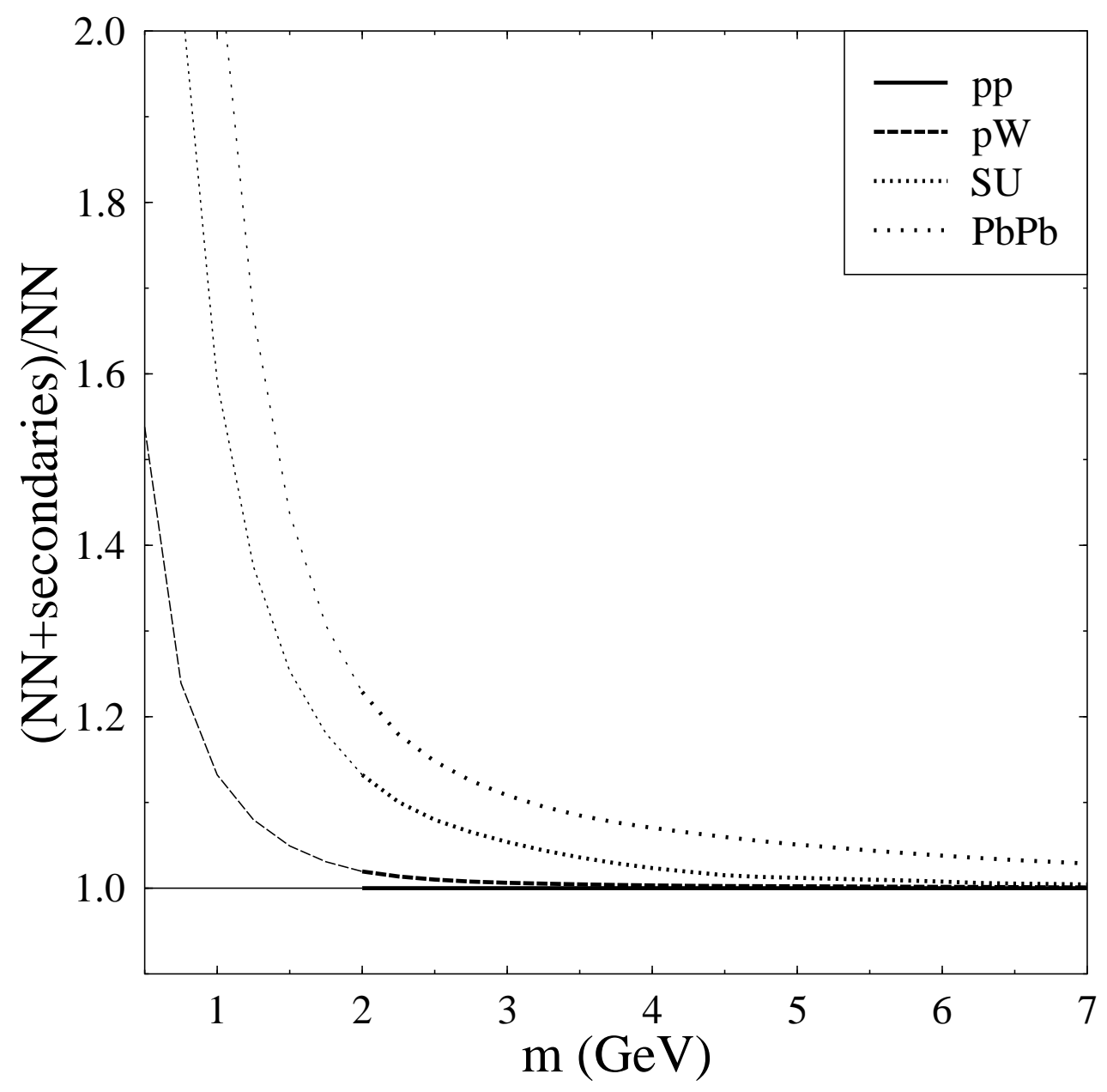

FIG. 5. Ratio between the total dilepton mass spectrum and the nucleon-nucleon scattering alone, at $y_{\mathrm{cms}}=0.5$, for different systems: $\mathrm{p}+\mathrm{W}$ and $\mathrm{S}+\mathrm{U}$ at $200 A \mathrm{GeV}, \mathrm{Pb}+\mathrm{Pb}$ at $160 A \mathrm{GeV}$. 
a)

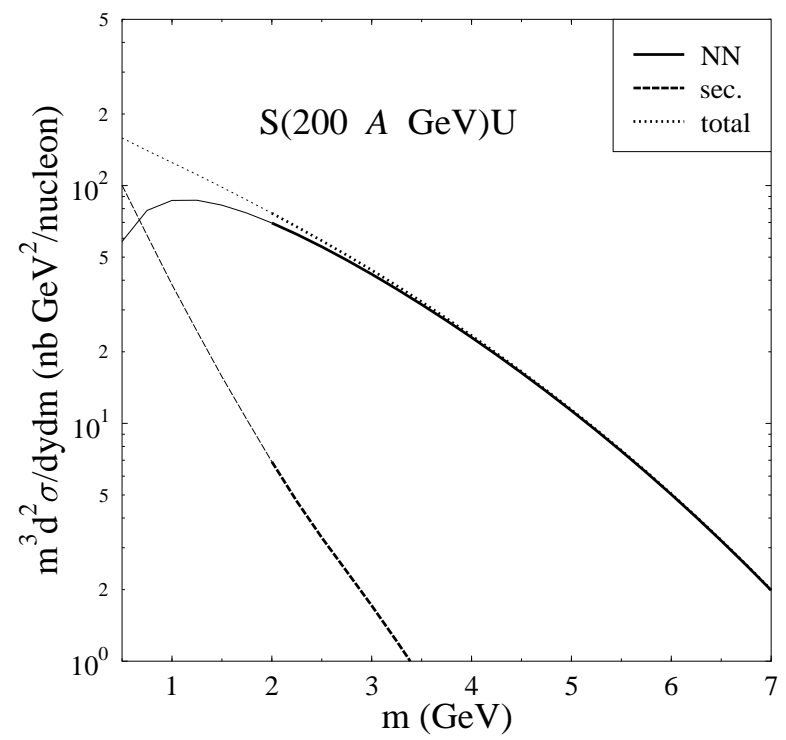

b)

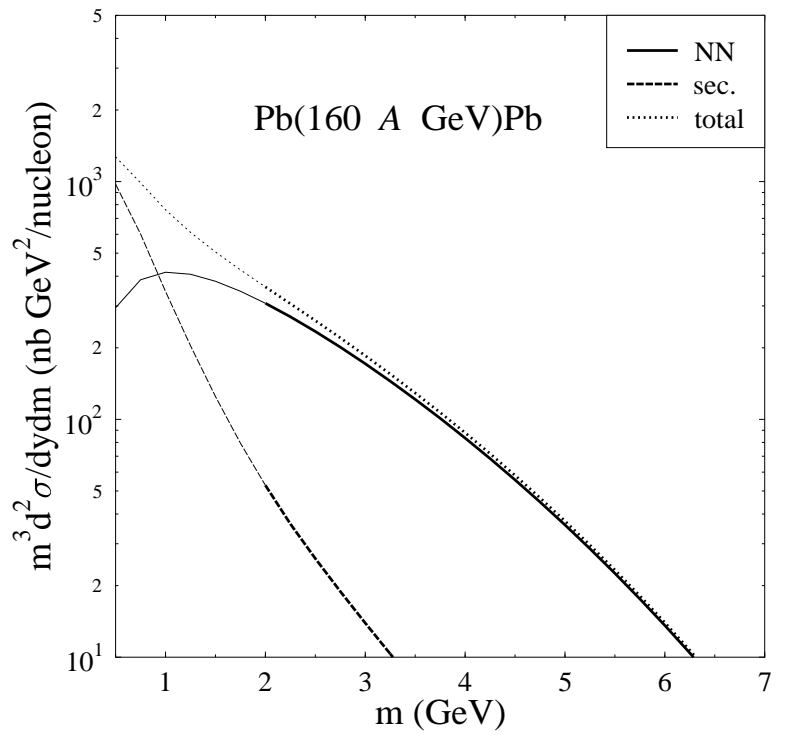

FIG. 6. Differential Drell-Yan cross section, per unit rapidity, at $y_{\mathrm{cms}}=0.5$, for the $10 \%$ most central collisions of $\mathrm{S}+\mathrm{U}$ at $p_{\text {lab }}=200 A \mathrm{GeV}$ (left) and of $\mathrm{Pb}+\mathrm{Pb}$ at $p_{\text {lab }}=160 A \mathrm{GeV}$ (right). The different Drell-Yan contributions from the hadronic simulation are shown. 
a)

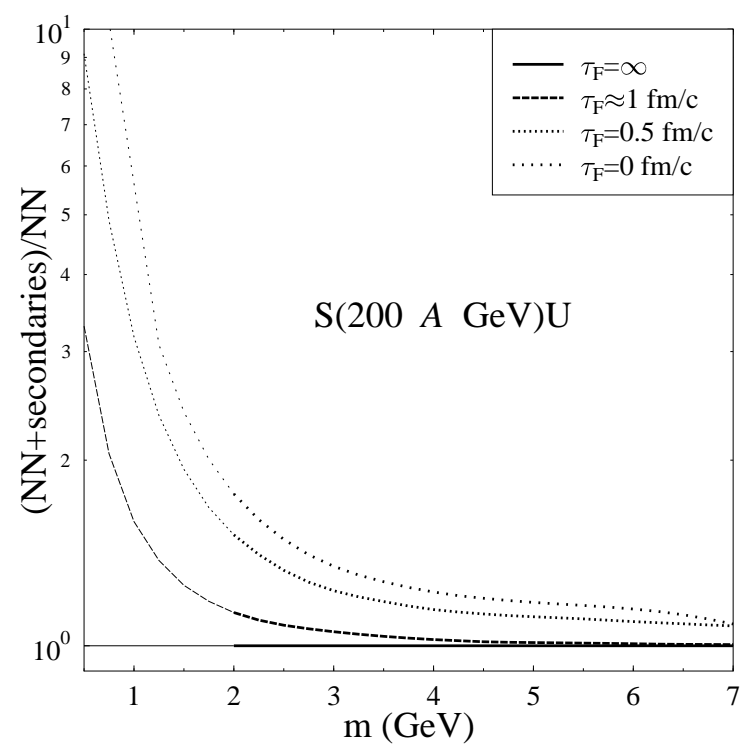

b)

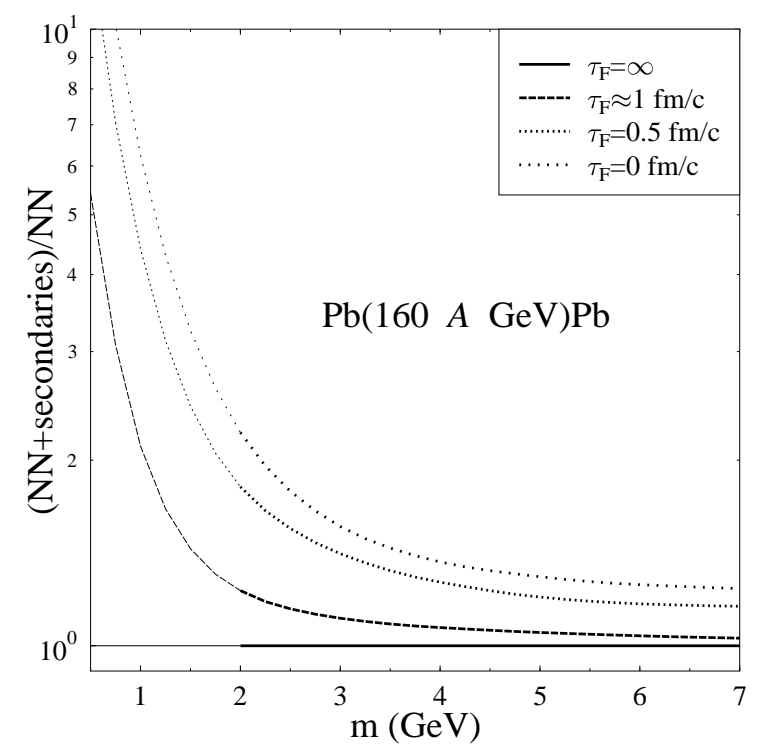

FIG. 7. Ratio between the total dilepton mass spectrum and the nucleon-nucleon scattering alone, at $y_{\mathrm{cms}}=0.5$, for $\mathrm{S}+\mathrm{U}$ colisions at $p_{\text {lab }}=200 A \mathrm{GeV}$ (left) and for $\mathrm{Pb}+\mathrm{Pb}$ collisions at $p_{\text {lab }}=160 A \mathrm{GeV}$ (right). The calculations correspond to different formation times for the secondary hadrons. 